LAWRENCE LIVERMORE N A TIO N A L LABORATORY
Charge-state distribution and Doppler effect in an expanding photoionized plasma

M.E. Foord, R.F. Heeter, R.S. Thoe, H.-K. Chung, D.A. Liedahl, W.H. Goldstein, P. T. Springer, J. E. Bailey, M.E. Cuneo, G.A. Chandler, L.P. Mix, P.A.M.van Hoof, C. Ramsbottom, R. Kisielius, V. Jonauskas, F.P. Keenan, S.J. Rose

March 3, 2004

Physical Review Letters 
This document was prepared as an account of work sponsored by an agency of the United States Government. Neither the United States Government nor the University of California nor any of their employees, makes any warranty, express or implied, or assumes any legal liability or responsibility for the accuracy, completeness, or usefulness of any information, apparatus, product, or process disclosed, or represents that its use would not infringe privately owned rights. Reference herein to any specific commercial product, process, or service by trade name, trademark, manufacturer, or otherwise, does not necessarily constitute or imply its endorsement, recommendation, or favoring by the United States Government or the University of California. The views and opinions of authors expressed herein do not necessarily state or reflect those of the United States Government or the University of California, and shall not be used for advertising or product endorsement purposes. 


\title{
Charge-state distribution and Doppler effect in an expanding photoionized plasma
}

\author{
M.E. Foord, R.F. Heeter, R.S. Thoe, H.-K. Chung, \\ D.A. Liedahl, W.H. Goldstein, and P.T. Springer \\ University of California, Lawrence Livermore National Laboratory. Livermore, CA 94551 \\ J.E. Bailey, M.E. Cuneo, G.A. Chandler, and L.P. Mix \\ Sandia National Laboratory, Albuquerque, NM, 37185 \\ P. A. M. van Hoof, C. Ramsbottom, R. Kisielius, V. Jonauskas, and F.P. Keenan, \\ Department of Pure and Applied Physics, \\ Queen's University Belfast, BTr 1NN, N. Ireland, UK \\ S.J. Rose \\ Department of Physics, Clarendon Laboratory, \\ Parks Road, Oxford OX1 3PU, UK
}

\begin{abstract}
The charge state distributions of $\mathrm{Fe}, \mathrm{Na}$ and $\mathrm{F}$ are determined in a photoionized laboratory plasma using high resolution x-ray spectroscopy. Independent measurements of the density and radiation flux indicate unprecedented values for the ionization parameter $\xi=20-25 \mathrm{erg} \mathrm{cm} \mathrm{s}^{-1}$ under near steady-state conditions. Line opacities are well fitted by a curve-of-growth analysis which includes the effects of velocity gradients in a one-dimensional expanding plasma. First comparisons of the measured charge state distributions with x-ray photoionization models show reasonable agreement.

PACS numbers: 52.50.Jm, 52.25.Kn
\end{abstract}


With the recent launch of the x-ray observatories Chandra and XMM-Newton, high resolution spectral data from numerous astrophysical x-ray sources such as accretion-driven binary systems and active galactic nuclei have been reported [1,2]. Discrete emission and absorption spectra from such objects are extremely complex. For example, in an x-ray binary system, the surrounding plasma is photoionized by the thermal radiation produced by the mass accretion from the companion star, generating observable emission and absorption spectra. Interpreting such spectra requires detailed modeling of the collisional and radiative processes involved as well as accurate atomic data. Laboratory studies, using for example electron-beam ion traps [3], storage rings [4], and tokamaks [5] have provided atomic data that have been incorporated into astrophysical spectral synthesis codes. However, until recently [6-10], creating an appropriate x-ray environment to carry out well characterized photoionization experiments under near steady-state conditions has not been possible, owing to the lack of a high fluence x-ray source. In this Letter, we present results from our Z-pinch experiments, where the x-ray emission from the pinch photoionizes Fe into the L-shell and $\mathrm{Na}$ and $\mathrm{F}$ into the $\mathrm{K}$ shell. Using high resolution $\mathrm{x}$-ray spectroscopy and other $\mathrm{x}$-ray diagnostics, the charge state distribution, the absolute radiative flux, and the sample densities are measured independently. These allow the first direct comparisons with photoionization models in the relatively low density $\left(\mathrm{n}_{\mathrm{e}}=2 \times 10^{19} \mathrm{~cm}^{-3}\right)$ two-body regime.

These experiments were performed at the Sandia National Laboratory Z-facility. The radiation from the pinch is generated by coupling a $20 \mathrm{MA}, 100 \mathrm{~ns}$ rise time current pulse into a $2 \mathrm{~cm}$ diameter, $1 \mathrm{~cm}$ length wire array, consisting of 300 tightly stretched $11.5 \mu \mathrm{m}$ diameter tungsten wires. The electromagnetic forces drive the wires radially inward onto the central axis, creating a 8 ns FWHM, 120 TW, 165 eV near-blackbody radiation source. An absolutely calibrated transmission grating spectrometer measures the spectral and temporal emission from the pinch [11] (see Fig. 1). The pinch emission provides sufficient flux at photon energies above $1 \mathrm{keV}$ to photoionize iron into the L-shell, and also produces a relatively line-free quasi-continuum source of X-rays in the $9-17 \AA$ wavelength range as needed for the absorption experiments described below [6,8-10]. Also shown in Fig. 1 are comparisons with filtered X-ray detectors (XRDs) [12] which show reasonable agreement over most of the energy range. Above $2 \mathrm{keV}$, spectra were measured using filtered PCD detectors [13]. The results presented below were found to be relatively insensitive to the small high energy component above $2 \mathrm{keV}$. 
Free standing thin $(500-750 \AA)$ rectangular foils were suspended in frames and positioned $1.5-1.6 \mathrm{~cm}$ from the z-axis of the pinch. The foils consisted of a 1.35:1 molar ratio of Fe/NaF and were overcoated on each side with $1000 \AA$ of lexan $\left(\mathrm{C}_{16} \mathrm{H}_{14} \mathrm{O}_{3}\right)_{n}$ to help maintain uniform conditions during heating and expansion. During the initial 100 ns run-in phase, radiation from the wires preheated the foils, and the central $\mathrm{Fe} / \mathrm{NaF}$ portion of the foil typically expanded 1.5 - $2 \mathrm{~mm}$, decreasing the density by many orders of magnitude. A time-gated filtered x-ray pinhole camera imaged the $\mathrm{Fe} / \mathrm{NaF}$ emission region edge-on, thus determining the time history of the average density. When the wires collide on axis, the resulting x-ray pulse quickly photoionizes the low density expanded foil.

In general, at sufficiently low density where two-body processes dominate, the charge state distribution is determined by a balance between photoionization and radiative and dielectronic recombination. In this regime, the degree of ionization can be shown to mainly depend on the ionization parameter $\xi \equiv 16 \pi^{2} J / n_{e}[14]$, where $J$ is the mean intensity, $J \equiv 1 / 4 \pi \iint J_{\nu}(\Omega) \mathrm{d} \Omega \mathrm{d} \nu$ integrated over solid angle and integrated in energy from $\chi_{H}=$ $13.6 \mathrm{eV}$ to infinity. $\xi$ is a commonly used scaling parameter in astrophysics which determines the photoionization state in low density astrophysical plasmas. Far from the radiation source, $\xi \simeq 4 \pi \Gamma / n_{e}$, where $\Gamma\left(\operatorname{erg~} \mathrm{cm}^{-2} \mathrm{~s}^{-1}\right)$ is the incident flux. In these experiments, near the peak of the radiation pulse when $\mathrm{n}_{e}=2 \times 10^{19} \mathrm{~cm}^{-3}, \xi$ reaches peak values near $25 \mathrm{erg} \mathrm{cm} \mathrm{s}{ }^{-1}$.

Quasi-continuum x-ray radiation produced by the pinch is absorbed as it passes through the photoionized plasma and is recorded on film using a time-integrated convex KAP crystal spectrometer. The foil was positioned vertically and expands along the line-of-sight between

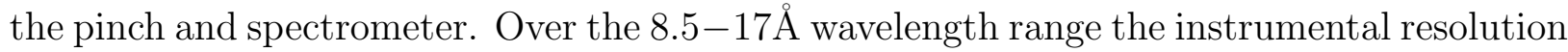
was $E / \Delta E \simeq 500-800$. Typical absorption data over the $8.5-12 \AA$ portion of the spectrum are shown in Fig. 2, where K-shell absorption lines for $\mathrm{Na}$ and $\mathrm{F}$ ions, and L-shell absorption lines for iron are observed.

The Na and $\mathrm{F}$ absorption lines were analyzed using a simple one-dimensional expansion model. Hydrodynamic simulations indicate that during the initial heating and expansion phase, the central $\mathrm{Fe} / \mathrm{NaF}$ portion of the foil remains fairly uniform due to the tamping effect of the lexan overcoat. The velocity profile is well approximated by the linear self-similar form $u(x)=u_{0}\left(x / x_{0}\right)$, where $u_{0}$ and $x_{0}$ are the velocity and position at the edge, relative to the foil center. The optical depth of a photon passing normal through this expanding one-dimensional plasma is given by: 


$$
\tau_{\nu}=\int_{-u_{0}}^{u_{0}} d u \frac{k_{0}}{\sqrt{\pi} u_{0} \sigma} \exp \left\{\frac{-\left[\nu\{1-u(x) / c\}-\nu_{0}\right]^{2}}{\sigma^{2}}\right\}
$$

where $\sigma$ is the transition line width dominated here by Doppler thermal motion (Stark broadening is small), $1-u(x) / c$ is the Doppler factor due to expansion, and $k_{0} \equiv$ $\pi e^{2} f_{\text {osc }} N_{i} L / 2 m c$, which depends on the oscillator strength of the line transition. $L \equiv 2 x_{0}$ and $N_{i} L$ is the areal density of the ground state configuration for ion $i$. Stimulated emission is small and is not included. Because the expansion is approximately one-dimensional and the ground-state populations dominate the total population of each ion stage, the areal density summed over each ion stage is conserved and can be written as $\sum_{i} N_{i} L=N_{o} L_{o}$, where $N_{o} L_{o}$ is the initial areal density of the solid foil (provided by the manufacturer). Thus, $N_{i} L=f_{i} N_{o} L_{o}$, where $f_{i}$ is the fractional charge state abundance of ion $i$.

Defining the new variables $y=\left(\nu-\nu_{0}\right) / \sigma$ and $w=y-u / u_{t h}$, using $u_{t h}=\sigma c / \nu_{0}$ and $u / c \ll 1$, and assuming that the sample density is approximately uniform, the optical depth is rewritten as:

$$
\tau_{\nu}=\frac{k_{0} u_{t h}}{\sigma u_{0}} \int_{y-u_{0} / u_{t h}}^{y+u_{0} / u_{t h}} \frac{\mathrm{e}^{-w^{2}}}{\sqrt{\pi}} d w=\frac{k_{0}}{2 \sigma} \frac{\left\{\operatorname{erf}\left(y+u_{0} / u_{t h}\right)-\operatorname{erf}\left(y-u_{0} / u_{t h}\right)\right\}}{u_{0} / u_{t h}}
$$

This expression reduces to a Gaussian profile having a thermal width $\sigma$ for the stationary case where $u_{0} / u_{t h} \ll 1$ and approaches a rectangular function for the case $u_{0} / u_{t h} \gg 1$.

In these experiments the instrumental resolution was insufficient to resolve the Doppler broadened line profiles in these relatively low temperature plasmas. However, the spectrally integrated line strength is independent of the instrumental resolution. Hence we define the measured line absorption strength (equivalent width) as $A=\int\left\{1-\exp \left(-\tau_{\nu}\right)\right\} d \nu$, where $\tau_{\nu}$ is given in Eq. 2. This yields a "curve of growth" relationship, often applied in interpreting astrophysical line strengths [15]

$$
\frac{A}{\sigma}=\int\left\{1-\exp \left(-\tau_{\nu}\right)\right\} d y=\int\left[1-\exp \left\{-\tau_{0} g(y)\right\}\right] d y
$$

where $\tau_{0}=2 k_{0} / \sqrt{\pi} \sigma$ is the unshifted line-center optical depth, and $g(y)$ is defined through Eq. 2. For optically thin lines where $\tau_{0}<1$, Eq. 3 predicts that the ratio of $A / \sigma$ is approximately proportional to $\tau_{0}$. In the optically thick regime, $A / \sigma$ depends on both $\tau_{0}$ and $u_{0} / u_{t h}$ since the center of the line starts to saturate. The transition from an optically thin linear regime to an optically thick non-linear regime at larger $\tau_{0}$ is evident in Fig. 
3. By fitting a range of data from relatively weak to strong absorption lines, the relative concentrations of each ion, $f_{i}$, can be determined.

The analysis is applied to the $\mathrm{Na}$ and $\mathrm{F}$ absorption spectrum shown in Fig. 2. The absorption strengths of the Na lines $1 s^{2}-1 s 2 p, 1 s^{2}-1 s 3 p, 1 s^{2}-1 s 4 p, 1 s^{2}-1 s 5 p$, and the $1 s-2 p_{1 / 2,3 / 2}$ doublet were determined by numerical integration across each respective absorption line. The absorption lines are broadened by both thermal effects and from the expansion of the plasma. Shown in Fig. 3 is a calculation assuming $T_{i}=150 \mathrm{eV}$ and $u_{0} / u_{t h}=0.83$. The data are well fitted by this distribution over a wide range of line strengths. The resulting ratio of $\mathrm{Na}^{10+}: \mathrm{Na}^{9+}$ (H-like:He-like) ground state ions is 1:4.5. We note that the charge state ratio of H-like to He-like ions depends mostly on the ratio of absorption line strengths and is relatively insensitive to the ion temperature. Similar analysis of $\mathrm{F}$ absorption lines indicates a ratio of $6.0: 1$ for $\mathrm{F}^{8+}: \mathrm{F}^{7+}$. The reversed ratio for F relative to $\mathrm{Na}$ is due to its lower photoionization threshold.

The Fe absorption spectrum was analyzed using the one-dimensional expansion model described above. Line positions and oscillator strengths for many thousands of Fe lines were calculated using the HULLAC suite of codes [16]. In some cases, line positions were modified to improve agreement with the measured line positions. In cases where nearby lines were strongly blended, more accurate line positions from Kelly [17] were used. The charge state distribution was then determined by varying each iron charge state concentration, $f_{i}$, to best fit the absorption line strengths. The resulting charge state distribution for Fe is shown in Fig. 4. Uncertainties in the background level and signal-to-noise noise of the absorption spectrum were used to estimate the uncertainties in the charge state populations for each ion. The average charge state for $\mathrm{Fe}$ is $\langle Z\rangle \simeq 16.1 \pm 0.2$.

The charge state distributions for $\mathrm{Fe}, \mathrm{Na}$, and $\mathrm{F}$ were calculated with the collisionalradiative code Galaxy[18]. For a given density, temperature, and incident radiation field, Galaxy calculates the steady-state ionization balance within the plasma. Collisional and radiative excitation and ionization, as well as autoionization and all reverse processes are included. A rate matrix is constructed that couples the initial and final levels using simple scaled-hydrogenic expressions. Accurate Hartree-Dirac-Slater(HDS) cross section are used for photoionization out of the ground state of arbitrary ionization stages for elements up to $Z=30$. For photoionization out of the excited levels, scaled HDS cross sections are used if the electron being removed is also present in the ground state, and Kramers cross sections are 
used otherwise. Corresponding radiative recombination rates are calculated using detailed balance. The Galaxy code employs an average-of-configuration approximation for electronic states with a principal quantum number $n \leq 5$ and averages over all the configurations with the same principal quantum number for higher $\mathrm{n}$.

In order to account for the estimated few ns required to reach steady-state equilibrium[19,20], the values of the absolute spectral flux and sample density $\left(n_{e}=2.0 \pm 0.7 \times\right.$ $10^{19} \mathrm{~cm}^{-3}$ ) used in the calculations were taken at $+3 \mathrm{~ns}$ after the peak of the radiation pulse. We estimate that the decrease in $\xi$ from 25 to $20 \mathrm{erg} \mathrm{cm} \mathrm{s}^{-1}$ during this time has a very small effect on the ionization balance. Using these values, the charge state distribution for Fe was calculated for various temperatures between $30 \mathrm{eV}$ and $210 \mathrm{eV}$ (see Fig. 4). Above 70 $\mathrm{eV}$, the distributions peak near $\mathrm{Fe}^{16+}$ and are quite insensitive to the electron temperature. In this temperature regime (90 to $210 \mathrm{eV}$ ) calculations indicate that photoionization of $\mathrm{Fe}$ L-shell ions dominates over collisional ionization processes by more than a factor of ten. The weak temperature dependence of the charge state distribution therefore is likely due to the thermal electrons having insufficient energy to ionize the L-shell ions in this regime. Below $50 \mathrm{eV}$, the contribution from three-body recombination begins to dominate, reducing the degree of ionization substantially.

The average charge state predicted by Galaxy in the 90 to $210 \mathrm{eV}$ temperature range is $<Z>\simeq 16.4 \pm 0.2$ (see Fig. 4). The uncertainty in $\langle Z\rangle$ is determined from folding in the sensitivities to the uncertainties in the absolute flux $( \pm 20 \%)$ and density $( \pm 35 \%)$ measurements. The calculated distribution is slightly more ionized than measured. This may be due, in part, to the fact that the measured time-integrated absorption spectrum is weighted by the time-history of the backlighter intensity, which peaks a few ns before the sample reaches steady-state equilibrium, resulting in a slightly lower average charge. Galaxy calculations of $\mathrm{H}$ to He-like ratios for $\mathrm{F}$ and $\mathrm{Na}$ yielded ratios of $6.7: 1$ and $1: 1.4$, respectively at $T_{e}=150 \mathrm{eV}$. The $\mathrm{F}$ ratio agrees well with the data $(6.0: 1)$, while the $\mathrm{Na}$ data $(1: 4.5)$ is somewhat less ionized than predicted.

For comparison, the photoionization code Cloudy[21] was also employed. Cloudy generally uses simplier atomic modeling than Galaxy but incorporates better atomic data and can also calculate the electron temperature independently. Cloudy calculates the temperature by a detailed energy accounting of all relevant heating and cooling processes in the plasma, such as from collisional excitation followed by radiative cooling. Our first model calculation 
included a restricted set of Fe emission lines that effectively treated the Fe resonance lines as optically thick. This assumption is consistent with the measured saturation of the strongest Fe lines and the curve of growth plots shown in Fig. 3. This model yielded an electron temperature near $T_{e}=150 \mathrm{eV}$ and an average charge state $\langle Z>\simeq 16.0$, in reasonable agreement with both the measured distribution width and the average ionization state (see Fig. 4). To test the sensitivity to optical depth effects, a second model was constructed which treated all lines as optically thin. This optically thin model yielded a much lower temperature $T_{e}=38$ $\mathrm{eV}$, due to enhanced cooling from line emission, and a distribution that peaked at $\mathrm{Fe}^{12+}$. The optically thin approximation is therefore inconsistent with the line absorption data and the resulting charge state distribution, but does set a lower limit on the temperature due to line transport. A more accurate calculation would require a self-consistent treatment of the line transport and population kinetics in an expanding geometry, which is beyond the scope of this work.

Also shown in Fig. 4 are model calculations from the collisional-radiative code Flychk. Details of this code are not discussed here, but are described in detail in Ref. 20. At $T_{e}=$ $150 \mathrm{eV}$, Flychk predicts a slightly higher average ionization $<Z>\simeq 16.5$. It is interesting to note that Flychk and Galaxy predict broader distributions than Cloudy. For example, for $T_{e}$ between $70 \mathrm{eV}$ and $210 \mathrm{eV}$, Galaxy predicts an average charge that varies between 16.2 to 16.4 and a FWHM that varies between 1.6 - 1.7, much broader than 1.2 for Cloudy. Differences in the widths are likely due to differences in the specific rates that couple the $\mathrm{Fe}^{+15}, \mathrm{Fe}^{+16}$, and $\mathrm{Fe}^{+17}$ ions. Understanding these differences between models, as well as including better atomic data where needed, such as R-matrix rates, is the subject of future work.

In this experiment we set out to produce a low density photoionized iron plasma in order to create conditions that would allow a meaningful comparison with x-ray emission from astrophysical sources. High resolution x-ray spectra of these sources are now, for the first time, becoming available through the Chandra and XMM-Newton missions. Analysis of these spectra is still hampered by a lack of well tested modeling codes and/or atomic data. We believe that these are the first laboratory experiments in which the charge state distribution was measured in a well characterized, strongly photoionized plasma where the ionization parameter reached values of $\xi=20-25 \mathrm{erg} \mathrm{cms}^{-1}$, an ionization regime relevant to astrophysical x-ray sources. The degree of ionization was found to be in reasonable agreement 
with the three photoionization codes tested, although differences in the distribution widths requires further study. We are currently considering ways to independently measure the electron temperature, such as through Thomson scattering[22] to allow direct comparisons with temperature predictions as well. We hope these results will also stimulate interest in future experiments at the ZR-facility upgrade and at the National Ignition Facility at LLNL, where even higher radiation fluxes are anticipated.

The authors thank T.R. Kallman, H. Netzer, G.J. Ferland, and R.W. Lee for helpful discussions, M.K. Matzen, A.L. Osterheld, and M. Eckart for their support, J. Emig and the Sandia Z-facility team for their technical assistance, and D. L. Fehl for assistance with XRD unfolds. This paper used the photoionisation code Cloudy, written by G.J. Ferland, which can be obtained from http://www.nublado.org. This work was performed under the auspices of the U.S. Department of Energy by the University of California Lawrence Livermore National Laboratory under contract No. W-7405-Eng-48. P.v.H. is supported by the Engineering and Physical Sciences Research Council of the United Kingdom. FPK and SJR acknowledge AWE Aldermaston for support through the William Penney Fellowship. This work was also supported by NATO Collaborative Linkage Grant CLG979443. 
[1] F. Paerels, et al., Astrophys. J. , 533, L135. (2000).

[2] J.S. Kaastra, J.S. Mewe, R., D.A. Liedahl, A.C. Brinkman, and S. Komossa, Astr. and Astro., 354, 83(2000).

[3] P. Beiersdorfer, et al., Astrophys. J. , 519, L185(1999).

[4] D.W. Savin, et al., Astrophys. J. , 489, L115 (1997).

[5] K.B. Fournier, et al., in Workshop on Data Needs for X-Ray Astronomy, eds. M.A. Bautista, T.R. Kallman and A.K. Pradhan, (Goddard Space Flight Center, Greenbelt, MD) p127 (2000).

[6] R.F. Heeter, et al.,ibid, p135 (2000).

[7] Y. Morita, et al., J. Quant Spect Rad 71529 (2001).

[8] R.F. Heeter, et al. Rev. Sci. Instr., 721224 (2001).

[9] M.E. Foord,et al. in Spectroscopic Challenges of Photoionized Plasmas ASP Conf. Series, eds. G.J. Ferland and D.W. Savin, 247 p117 (2001).

[10] J.E. Bailey, et al., Phys. Plasmas,9, 2186 (2002).

[11] L.E. Ruggles, et al., Rev. Sci. Instr., 72, 1218 (2001).

[12] G.A. Chandler,et al., Rev Sci. Instr.,70, 561(1999).

[13] T.W.L. Sanford, et al., Rev. Sci. Instr.,68, 852 (1997).

[14] C.B. Tarter, W.H. Tucker, and E.E. Salpeter, Astrophys. J. ,156, 943 (1969).

[15] D. Mihalas, Stellar Astrophysics, Freeman and Co. eds., San Francisco (1978).

[16] M. Klapisch, J. Schwob, B. Fraenkel, J. Oreg, J Opt Soc Am, 67,148,(1977).

[17] R.L. Kelly, J of Phys and Chem Ref Data, 16, (Suppl. No. 1) (1987).

[18] S.J. Rose, J.Phys B: At. Mol. Opt. Phys., 31, 2129 (1998).

[19] H.-K. Chung, private communication (2003).

[20] H.-K. Chung, W. L. Morgan and R. W. Lee, J of Quant Spect Rad Trans, 80, 107 (2003).

[21] G.J. Ferland, et al., PASP 110, 776 (1998).

[22] M.E. Foord,et al., Phys Rev Lett,85, 992 (2000). 


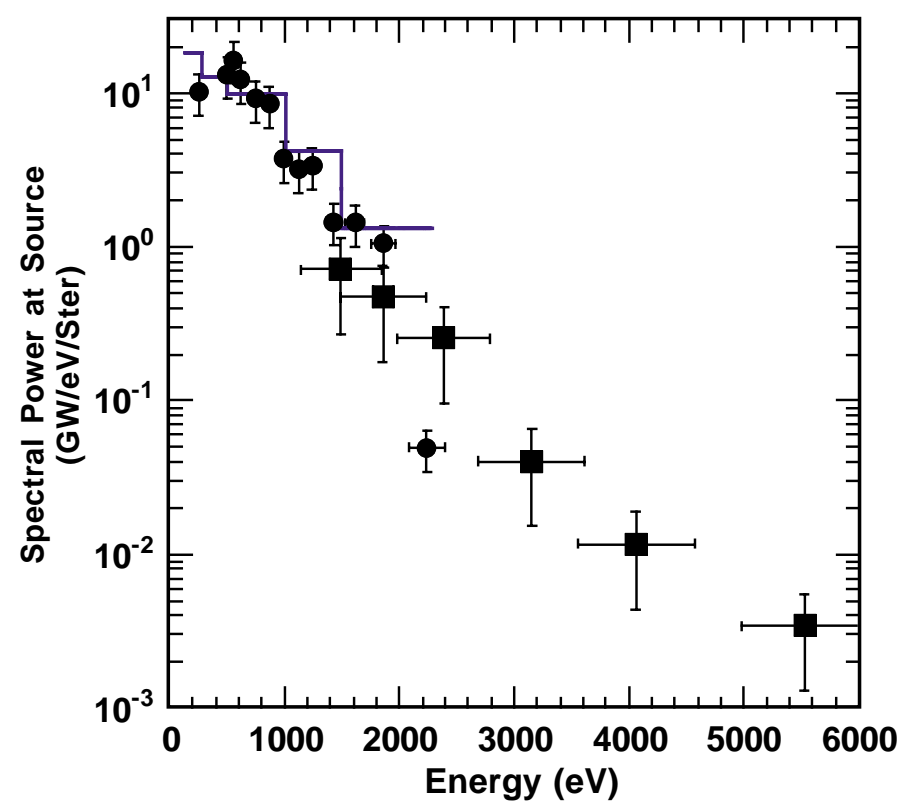

FIG. 1: Z-pinch x-ray spectral emission measured at peak power with an XRD array (solid line), a transmission grating spectrometer (dots), and a PCD array (squares).

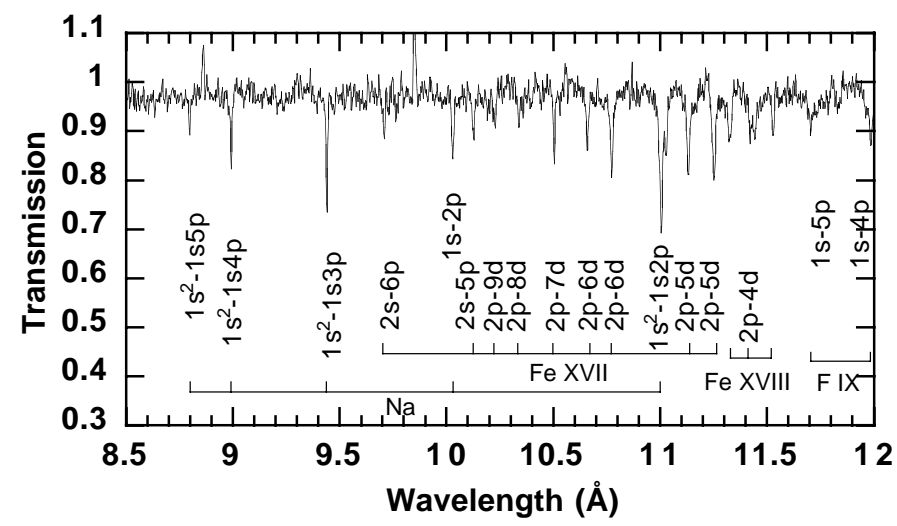

FIG. 2: Absorption spectrum of L-shell iron and K-shell sodium and fluorine lines. 


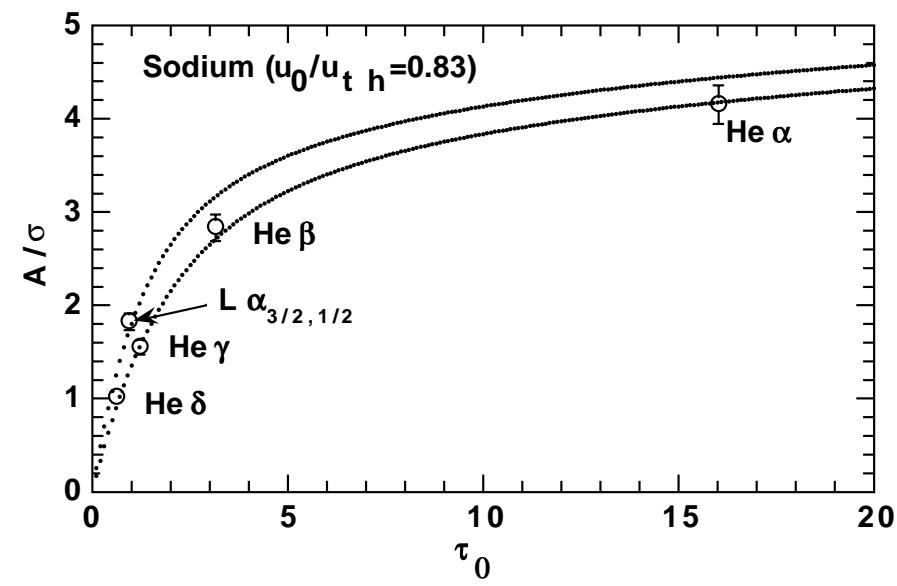

FIG. 3: Curve-of-growth analysis for sodium absorption lines. Vertical error bars assume $\pm 5 \%$ uncertainty in the absorption strength determined by numerically integrating the film spectrum for each line. The upper curve was calcalated for the blended $L y_{\alpha(3 / 2,1 / 2)}$ lines separated by $6 \mathrm{~m} \AA$.

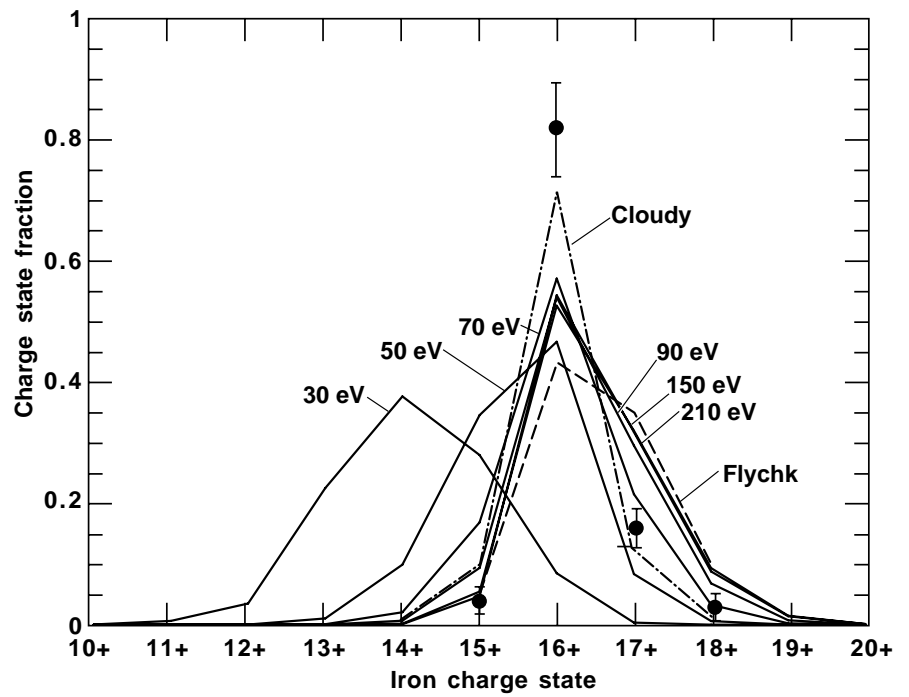

FIG. 4: Iron charge state distribution (solid dots) and comparisons with photoionization code Galaxy for $T_{e}=30 \mathrm{eV}-210 \mathrm{eV}$ (solid lines). Also shown are results from the code Flychk at $T_{e}$ $=150 \mathrm{eV}$ (dashed line) and Cloudy (dash-dot). The discrete fractional abundances are connected with lines to aid in viewing. 